\title{
SIMILARITY CRITERIA FOR PROCESSES OF GRAVITATIONAL CLASSIFICATION
}

\author{
E. BARSKY ${ }^{\mathrm{a}, *}$ and M. BARSKY ${ }^{\mathrm{b}}$ \\ ${ }^{a}$ Department of Industrial Engineering, Sami Shamoon (formerly Negev), \\ College of Engineering, Beer-Sheva, Israel; ${ }^{\mathrm{b}}$ Institutes for Applied Research \\ Ben-Gurion University of the Negev, Beer-Sheva, Israel
}

(Received 17 August 2004; In final form 2 September 2004)

\begin{abstract}
Similarity criteria for processes such as gravitational separation of bulk materials are understood as relationships that make it possible to obtain universal curves of separation. For turbulent separation regimes, such criteria were determined earlier [E. Barsky and M. Barsky, Master Curve of Separation Processes. Phys. Sep. Sci. Engin., 13 (2004), 1-13; M.D. Barsky, Powder Fractionation, Moscow, Nedra, 1980 (in Russian)]. In the present article, on the basis of cascade and structural model, similarity criteria are developed, which make it possible to obtain universal curves of separation in all regimes of the motion of the medium. Their applicability is demonstrated on concrete experimental data.
\end{abstract}

Keywords: Similarity criteria; Gravitational separation; Universal curves of separation; Cascade classifier

\section{INTRODUCTION}

The proof of the universality of fractional separation curves for processes occurring in the regimes of strong turbulence can be considered as a serious achievement in the development of the theory of gravitational processes [1,2].

Fractional separation of a narrow particle size class implies the relationship:

$$
F_{f}(x)=\frac{r_{f}}{r_{s}} \gamma_{f}
$$

where $r_{f}$ is the content of a narrow size class in the fine product outlet; $r_{s}$ is the content of the same narrow class in the initial material supplied for separation, $\% ; \gamma_{f}$ is the yield in the fine product outlet, $\%$.

The separation curves represent graphic dependences of the type:

$$
F_{f}(x)=f(w)
$$

*Corresponding author. E-mail: barskym@bgumail.bgu.ac.il 
for a fixed narrow size class where particle size $d=$ const. or:

$$
F_{f}(x)=\varphi(d)
$$

for a fixed-flow velocity ( $w=$ const $)$.

The universality of these curves rests on the fact that at a respective transformation of the abscissa axis, all separation curves obtained in a concrete classifier, for different narrow size classes, and different flow rates, are transformed into one curve. It simplifies considerably the computation of the process results, its optimization and allows absolutely unbiased comparison of different separation apparatuses. For the transitional and the laminar regimes of the motion of the medium, no parameters suitable for the generalization of separation curves have been found yet. Such parameters are extremely important for developing the theory and applications of gravitational separation of bulk materials.

\section{UNIVERSALITY OF SEPARATION CURVES}

We can illustrate the properties of the separation curves by specific examples. A set of experiments on fractionation of a coarse-grained material, chromium oxide, was carried out in an air cascade classifier with pour-over shelves (Fig. 1) at the concentration of the material $2.0-2.2 \mathrm{~kg} / \mathrm{m}^{3}$. The separation was carried out in an apparatus with five

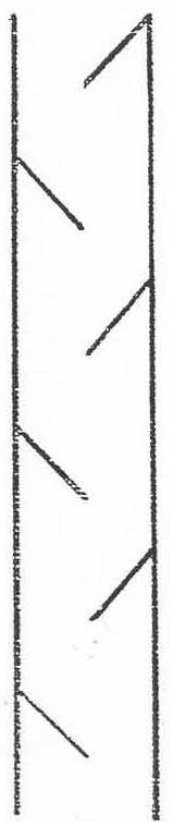

(a)

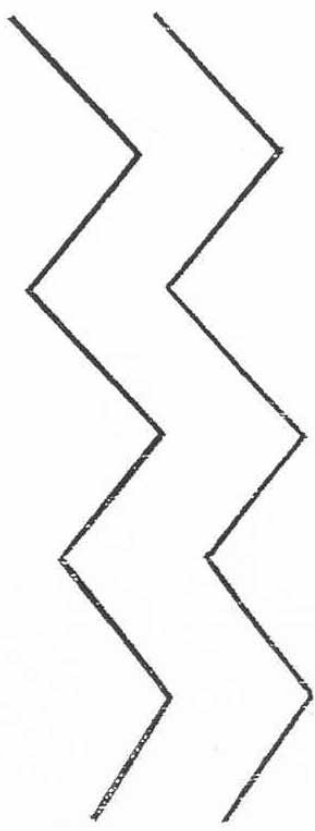

(b)

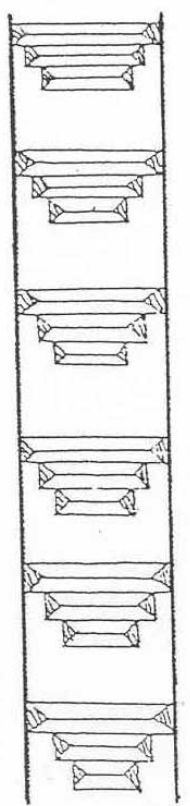

(c)

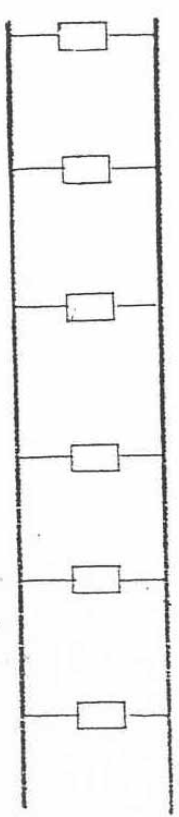

(d)

FIGURE 1 Several types of cascade classifiers: (a) with re-pouring shelves, (b) zigzag, (c) polycascade, (d) with radial grates. 
cascade stages $(z=5)$, at the initial feeding to the third shelf, counting top-down $\left(i^{*}=3\right)$. The grain-size distribution of the initial material is presented in Table I.

The experiments were carried out at the air flow rates equal to $2.7 ; 3.0 ; 3.25 ; 4.15$; $4.75 \mathrm{~m} / \mathrm{s}$.

As a summarizing criterion, in this case we use the expression previously obtained [1]:

$$
B=\frac{g d}{w^{2}} \frac{\left(\rho-\rho_{0}\right)}{\rho_{0}},
$$

where $g$ is the gravitational acceleration $\left(\mathrm{m} / \mathrm{s}^{2}\right), d$ is the particle size $(\mathrm{m}), w$ is the flow velocity $(\mathrm{m} / \mathrm{s}), \rho$ is the solid particles density $\left(\mathrm{kg} / \mathrm{m}^{3}\right)$ and $\rho_{0}$ is the density of the moving medium $\left(\mathrm{kg} / \mathrm{m}^{3}\right)$.

It can be easily shown that this criterion is dimensionless. Figure 2 represents the graphical dependence:

$$
F_{f}(x)=\varphi(B)
$$

\begin{tabular}{|c|c|c|c|c|c|c|c|c|c|c|c|c|}
\hline $\begin{array}{l}\text { Average particle size, } \\
\qquad d(\mathrm{~mm})\end{array}$ & 0.025 & 0.0565 & 0.0815 & 0.13 & 0.18 & 0.258 & 0.358 & 0.515 & 0.815 & 1.3 & 2.05 & 3.75 \\
\hline $\begin{array}{l}\text { Narrow class } \\
\text { content } r_{i}, \%\end{array}$ & 10.7 & 4.3 & 5.9 & 6.9 & 4.1 & 9.7 & 7.1 & 11.4 & 11.2 & 11.1 & 14.3 & 3.2 \\
\hline
\end{tabular}

TABLE I Granulometric composition of chromium oxide

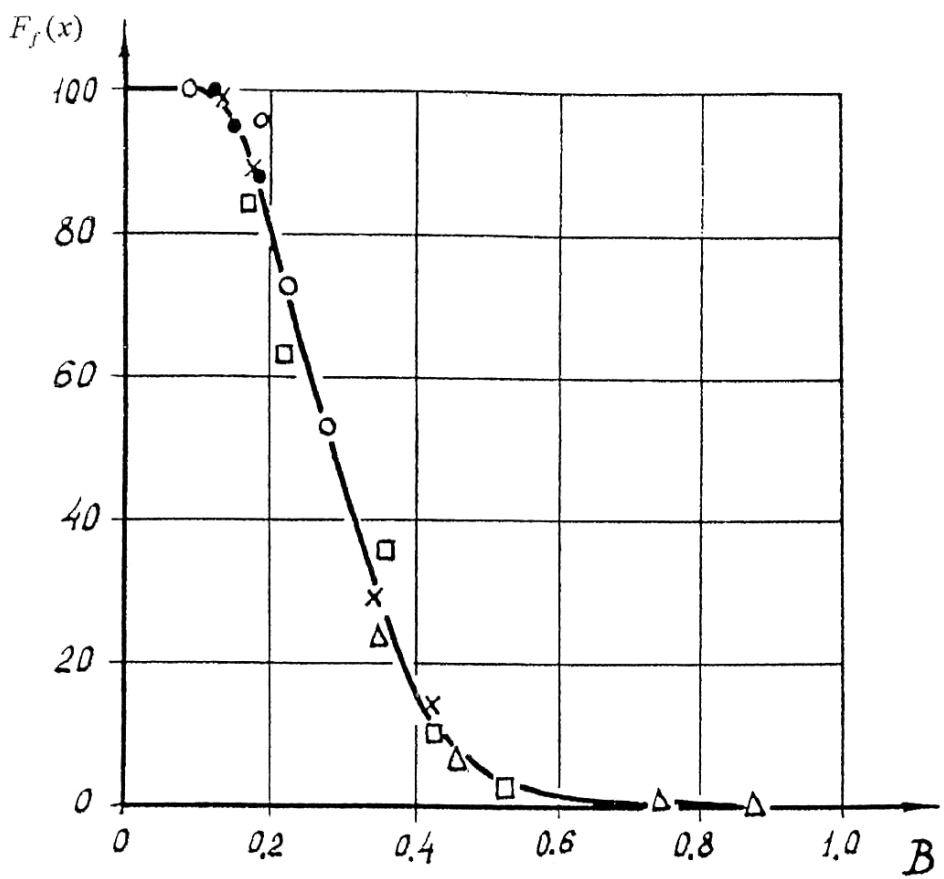

FIGURE 2 Dependence of fractional separation on the parameter $B$ for chromium oxide $\mathrm{Cr}_{2} \mathrm{O}_{3}$. Notation of the mean particle size: $\bullet-0.13 \mathrm{~mm} ; \bigcirc-0.258 \mathrm{~mm} ; \times-0.51 \mathrm{~mm} ; \square-0.815 \mathrm{~mm} ; \Delta-2.05 \mathrm{~mm}$. 
obtained on the basis of experiments carried out at all flow velocities and with all narrow classes of particles. Proceeding from this figure, we can conclude that in this case the parameter given by Eq. (4) makes it possible to obtain a universal curve.

As another example, we examine separation of a fine-grained material, aluminum powder, on a cascade multiple-shelf apparatus comprising 10 stages $\left(z=10 ; i^{*}=5\right)$ at the concentration of the material $1.5-2.2 \mathrm{~kg} / \mathrm{m}^{3}$. Grain size distribution of the aluminum powder is presented in Table II.

The experiments were carried out at the air flow velocities equal to $0.27 ; 0.36 ; 0.51$; $0.9 ; 1.32 ; 1.7 \mathrm{~m} / \mathrm{s}$.

A similar dependence:

$$
F_{f}(x)=\varphi(B)
$$

for this set of experiments is presented in Fig. 3.

Hence, in this case the parameter $B$ does not ensure the derivation of a universal curve. The reason for this conclusion rests, apparently, in the fact that in the first case the turbulent overflow of particles takes place, whereas in the second case the overflow of smaller particles at lower flow velocities occurs in a laminar or transitory

TABLE II Granulometric composition of aluminum powder

\begin{tabular}{lcccccccccc}
\hline $\begin{array}{l}\text { Average particle } \\
\text { size, } d(\mu \mathrm{m})\end{array}$ & 10 & 25 & 40 & 56.5 & 71.5 & 90 & 112.5 & 142.5 & 180 & $>180$ \\
Class content $r_{i}, \%$ & 3.6 & 7.2 & 17.0 & 8.2 & 7.8 & 6.7 & 6.5 & 7.5 & 7.8 & 27.7 \\
\hline
\end{tabular}

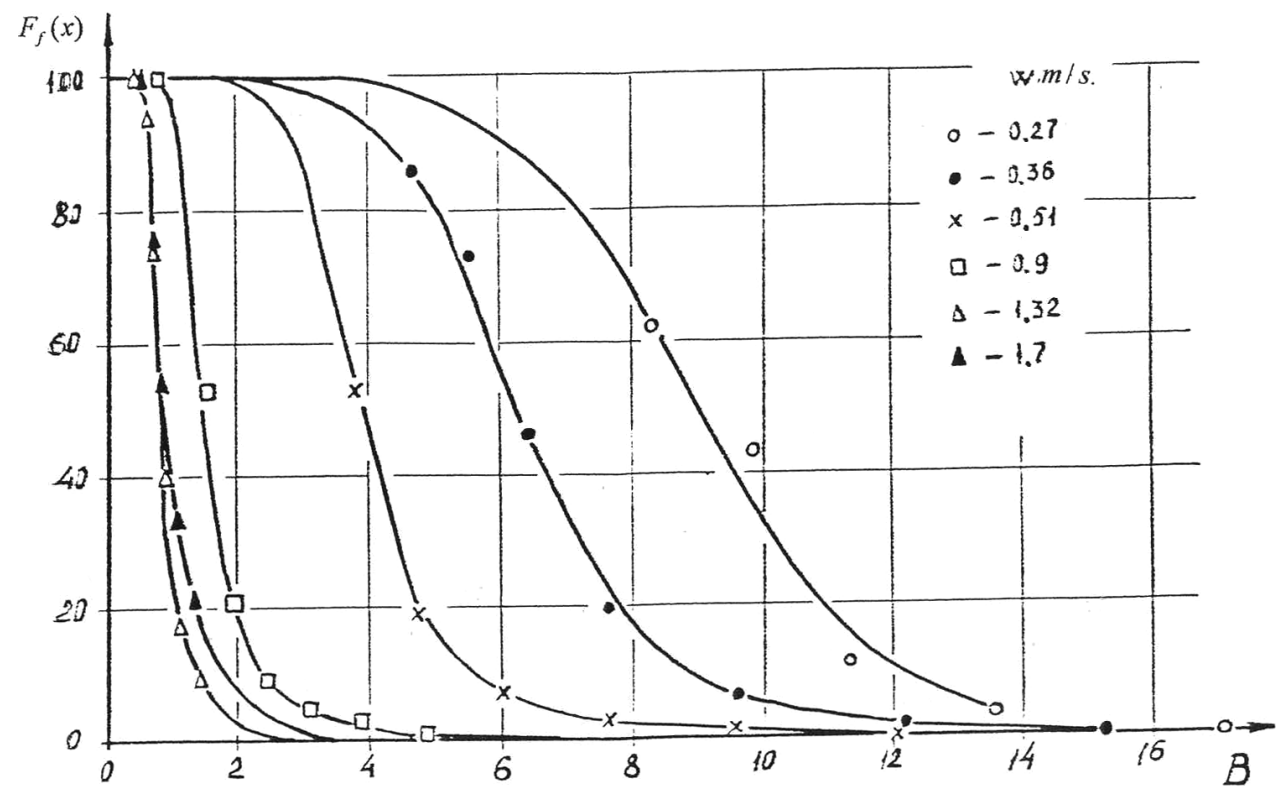

FIGURE 3 Dependence of fractional separation on the parameter $B$ for aluminum powder. Notation of the velocities: $\bigcirc-0.27 \mathrm{~m} / \mathrm{s} ; \bullet-0.36 \mathrm{~m} / \mathrm{s} ; \times-0.51 \mathrm{~m} / \mathrm{s} ; \square-0.9 \mathrm{~m} / \mathrm{s} ; \Delta-1.32 \mathrm{~m} / \mathrm{s} ; \boldsymbol{\Delta}-1.7 \mathrm{~m} / \mathrm{s}$. 
regimes. Therefore, we can make an attempt to find generalizing parameters from the standpoint of structural and cascade models of the process for these modes, as well.

\section{CASCADE MODEL OF SEPARATION}

Any vertical apparatus can be assumed to consist of a certain number of stages with a directional mass exchange of particles among them. A cascade classifier consisting of structurally similar or different stages gives the simplest idea of the stepwise character of the process.

The value characterizing narrow grade size exchange at a separate stage can be represented in the form [2]:

$$
K=\frac{r_{i}^{*}}{r_{i}}
$$

where $r_{i}$ is the original content of narrow particle size class at a certain $i$ th stage of the apparatus; $r_{i}^{*}$, the number of such particles passing from the $i$ th stage to the upper $(i-1)$ th stage; and $K$, the separation coefficient.

A general scheme of particle distribution over the height of the apparatus while feeding to the $i^{*}$ th stage is shown in Fig. 4.

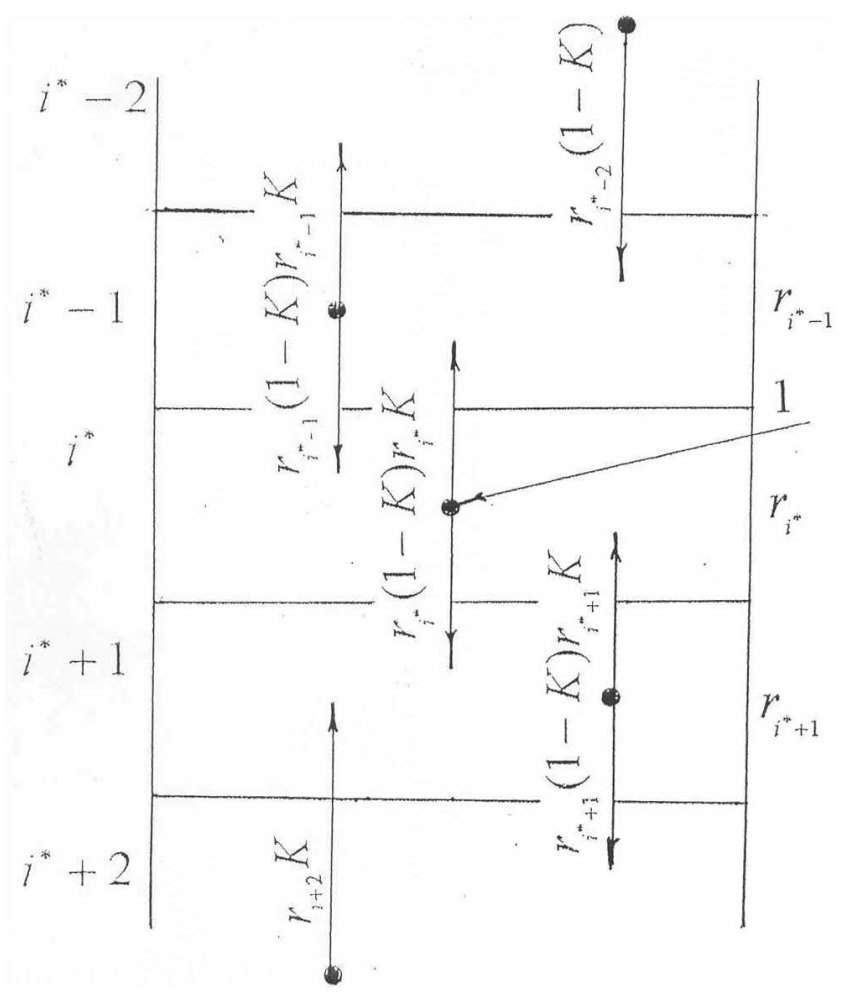

FIGURE 4 Schematic diagram of a narrow class separation inside a cascade apparatus. 
We take the initial content of one narrow class particles as a unity and assume that it is mixed with other particles when it is being fed into the separator.

It was proved in articles $[2,3]$ that for any size grade $j$, the fractional extraction into the fine product is described by the function:

$$
F_{f}\left(x_{j}\right)=\left[\begin{array}{cl}
\frac{1-\chi^{z+1-i^{*}}}{1-\chi^{z+1}} ; & K \neq 0,5 ; k \neq 0 \\
\frac{z+1-i^{*}}{z+1} ; & K=0,5 \\
0 ; & K=0
\end{array}\right.
$$

where $\chi=(1-K / K)$; and $i^{*}$ is the number of the stage onto which the material is being fed into the apparatus.

Under fixed unambiguity conditions $\left(z ; i^{*}\right)$, fractional separation for the entire apparatus is determined only by the value of the separation coefficient $K$. This is valid for the separation of coarse-grain powders in turbulent flows.

The dependence expressed by Eq. (7) can become fundamental for the computation of equilibrium and cascade classifiers, if we can define how the parameter $K$ is formed depending on the operating features of the process.

\section{STRUCTURAL MODEL OF THE PROCESS}

In the course of the development of the structural model, a number of assumptions for the separation coefficient were made:

(a) Particles shape is spherical;

(b) Distribution of particles of any narrow size grade over the apparatus cross section is uniform due to their intense interaction with each other, as well as with the apparatus walls and internal facilities.

Distribution of the local velocities of a continuous phase is a certain function of the geometrical characteristics of the channel feed cross section. It can be written in the general form as follows:

$$
u_{r}=w \cdot f\left(\frac{r}{R}\right)
$$

where $f(r / R)$ is a certain function associated with the cross-section geometry; $r$, the characteristic coordinate of a certain point of the apparatus cross section; $R$, the characteristic dimension of the apparatus cross section; $u_{r}$, local velocity of the continuous phase at the point with the coordinate $r ; w$, mean flow velocity.

Therefore, the dependence (8) takes into account the channel cross-section shape.

According to Newton-Rittinger's law, the dynamic effect of the flow on a single particle is determined by the dependence:

$$
F_{r}=\lambda \frac{\pi d^{2}}{4} \rho \frac{\left(u_{r}-v_{r}\right)}{2}
$$


where $F_{r}$ is the aerodynamic resistance, $\lambda$, the drag coefficient of a particle; $\left(\pi d^{2} / 4\right)$, the cross-sectional area of a particle, $\rho$, the flow density; $v_{r}$, the local absolute velocity of the particles and $\left(u_{r}-v_{r}\right)$, the particle velocity, with respect to the flow.

The flow direction is taken as the positive direction of the velocities $u_{r}$ and $v_{r}$.

If we take the total number of particles of a given mono-fraction in the cross section under study as equal to unity, the separation coefficient can be written as $K=n$ relative number of particles of a given narrow size grade with absolute velocity equal or greater than zero $(v \geq 0)$.

Examining the equilibrium of an isolated particle at a distance $r_{0}$ from the axis, we obtain:

$$
\frac{\pi d^{3}}{6} q\left(\rho-\rho_{0}\right)=\lambda \frac{\pi d^{2}}{4} \rho_{0} \frac{\left(u_{r}-v_{r}\right)^{2}}{2}
$$

hence,

$$
u_{r}-v_{r}=w \sqrt{\frac{4}{3 \lambda} \cdot B}
$$

Let us examine the mode of turbulent overflow of a particle characterized by a constant drag coefficient $\lambda$. In this case, the Reynolds number for the particle amounts to:

$$
\operatorname{Re}=\frac{\left(u_{r}-v_{r}\right) d \rho_{0}}{\mu} \geq 500
$$

where $\mu$ is the dynamic viscosity of a medium.

Taking into account Eq. (11), we obtain:

$$
\frac{w \sqrt{(4 / 3 \lambda) \cdot B} \cdot d \rho_{0}}{\mu} \geq 500
$$

This condition corresponds to the expression (for $\lambda=0,5$ ):

$$
\sqrt{\frac{8}{3} A r} \geq 500
$$

where $A r$ is the Archimedes number:

$$
A r=\frac{q d^{2} \cdot \rho \cdot \rho_{0}}{\mu^{2}}
$$

Hence, we can determine the limiting size of particles, above which their overflow is certainly turbulent. 
Let us consider the peculiarities of the laminar overflow of particles. In this case, the following relationship is valid:

$$
\operatorname{Re}=\frac{\left(u_{r}-v_{r}\right) d \rho_{0}}{\mu} \leq 1
$$

It follows from the equilibrium conditions that:

$$
\frac{\pi d^{3}}{6} q \rho=3 \pi \mu\left(u_{r}-v_{r}\right) d
$$

Taking the above-stated into account, an expression for $A r$, in this case, can be written as:

$$
A r=18 \mathrm{Re}, \quad A r \leq 18
$$

As is well-known, in the laminar overflow, the drag coefficient of particles can be written as:

$$
\lambda=\frac{24}{\operatorname{Re}}=\frac{24 \mu}{\left(u_{r}-v_{r}\right) d \rho_{0}}
$$

The following relationship can be derived from Eq. (11):

$$
\frac{u_{r}-v_{r}}{w}=\frac{1}{18} \operatorname{Re} B
$$

where $\mathrm{Re}$ is the Reynolds number calculated assuming the mean flow velocity.

Taking into account the equality $\operatorname{Re}^{2} B=A r$, we obtain:

$$
\frac{u_{r}-v_{r}}{w}=\frac{\sqrt{A r \cdot B}}{18}
$$

where $\operatorname{Re}=\left(d w \rho_{0} / \mu\right)$ is the Reynolds number.

Taking into account Eq. (11), we can write, for particles of a narrow class with an absolute velocity $v_{r} \geq 0$ :

$$
u_{r} \geq w \sqrt{\frac{4 B}{3 \lambda}}
$$

By introducing this relation into Eq. (8), we obtain:

$$
f\left(\frac{r}{R}\right) \geq \sqrt{\frac{4}{3 \lambda} \cdot B}
$$

Similarly, for particles with $v_{r} \leq 0$ :

$$
f\left(\frac{r}{R}\right) \leq \sqrt{\frac{4 B}{3 \lambda}}
$$


The inequalities in Eqs. (21) and (22) include the following limiting cases:

(1) for any coordinate:

$$
f\left(\frac{r}{R}\right)>\sqrt{\frac{4 B}{3 \lambda}},
$$

in this case, the separation coefficient is $K=1$;

(2) for $f(r / R)<\sqrt{4 B / 3 \lambda}$ we obtain $K=0$ for any coordinate $r$.

The intermediate case is characterized by the fact that some coordinates form level lines according to equality:

$$
f\left(\frac{r_{0}}{R}\right)=\sqrt{\frac{4 B}{3 \lambda}}
$$

We assume that this equation has one real root:

$$
\frac{r_{0}}{R}=f^{-1}\left(\frac{4 B}{3 \lambda}\right)
$$

Taking this into account, we find the respective area $\omega_{r_{0}}$ (see Fig. 5), for which:

$$
f\left(\frac{r_{i}}{R}\right) \geq f\left(\frac{r_{0}}{R}\right)
$$

is valid. Then the separation coefficient can then be written as

$$
\begin{aligned}
& K=\frac{\omega_{r_{0}}}{\omega_{R}}=C\left(\frac{r_{0}}{R}\right) \quad \text { for the convex profile of } f\left(\frac{r}{R}\right), \\
& K=C\left[1-\left(\frac{r_{0}}{R}\right)\right] \text { for the concave profile of } f\left(\frac{r}{R}\right) .
\end{aligned}
$$

The coefficient $C$ characterizes the form of level lines and feed-through cross section. For instance, for a circle $C=1$. By plugging the dependence (21) into the derived equations, we finally obtain:

$$
K=\varphi\left(\frac{B}{\lambda}\right)
$$

A similar dependence is valid for two and more roots of Eq. (7), which takes place in the case of complicated profiles $f(r / R)$. Thus, in case of profile shown in Fig. 5, for a certain mono-fraction, we obtain three real roots of Eq. (22): $r_{0_{1}} ; r_{0_{2}} ; r_{0_{3}}$. These roots form isotaches with the shape of the feed-through cross section of the apparatus taken into account. The isotaches determine corresponding total $\sum \omega_{r_{0 i}}$, for which the following relationship is valid:

$$
f\left(\frac{r}{R}\right) \geq f\left(\frac{r_{0 i}}{R}\right)
$$


Therefore, in the case under consideration:

$$
\sum \omega_{r_{0 i}}=\omega_{r_{01}}+\omega_{r_{02}}+\omega_{r_{03}}
$$

and the separation coefficient can be written as:

$$
K=\frac{\sum \omega_{r_{0 i}}}{\omega_{R}}
$$

Since $\sum \omega_{r_{0 i}}$ is unambiguously expressed through $r_{0 i}$, representing roots of Eq. (22), the final expression for the separation coefficient is:

$$
K=\varphi\left(\frac{B}{\lambda}\right)
$$

A specific expression for the separation coefficient can be obtained passing to a specific profile of the continuous medium diagram over the apparatus cross section. We consecutively examine possible cases of the interaction of particles in a flow.

\section{TURBULENT OVERFLOW OF PARTICLES AND TURBULENT REGIME OF THE MEDIUM MOTION IN THE APPARATUS}

In this case, for an equilibrium apparatus with a circular cross section, the distribution of the continuous medium velocities over the radius is usually expressed by the following empirical dependence [4]:

$$
u_{r}=w \frac{(n+1)(n+2)}{2} \cdot\left(1-\frac{r}{R}\right)^{n}=w f\left(\frac{r}{R}\right)
$$

where $n$ is an exponent depending on the mode of the medium motion and on the wall roughness, $(n<1)$.

According to Eq. (23), we can find the coordinate of isotach, where the absolute velocity of a fixed mono-fraction equals zero, i.e. for hovering conditions:

$$
\frac{(n+1)(n+2)}{2} \cdot\left(1-\frac{r_{0}}{R}\right)^{n}=\sqrt{\frac{4 B}{3 \lambda}}
$$

whence

$$
\frac{r_{0}}{R}=1-\left[\frac{2}{(n+1)(n+2)} \sqrt{\frac{4 b}{3 \lambda}}\right]^{1 / n}
$$

The cross-sectional area for which:

$$
f\left(\frac{r}{R}\right) \geq f\left(\frac{r_{0}}{R}\right)
$$

is equal to $\omega_{r_{0}}=\pi r_{0}^{2}$. 
Consequently, the separation coefficient is expressed by the ratio:

$$
K=\left(\frac{r_{0}}{R}\right)^{2}
$$

Then, taking into account Eq. (31), we obtain:

$$
K=\left\{1-\left[\frac{2 \cdot \sqrt{(4 B / 3 \lambda)}}{(n+1)(n+2)}\right]^{1 / n}\right\}^{2}
$$

Instead of the dependence in Eq. (29), we can examine other profiles of the distribution of continuous medium velocities over the radius:

$$
u_{r}=\frac{n+2}{n} \cdot w\left[1-\left(\frac{r}{R}\right)^{n}\right]
$$

where $n$ is the degree of flow turbulence $(n=2 \div \infty)$.

We shall examine the following cases:

(1) The velocity gradient on the flow axis.

For the dependence in Eq. (35) the following relation is valid:

$$
\frac{d u}{d r}=-(n+2) \cdot \frac{w}{R}\left(\frac{r}{R}\right)^{n-1}
$$

For the dependence in Eq. (31) we have:

$$
\frac{d u}{d r}=-\frac{w}{R} \cdot \frac{n(n+1)(n+2)}{2(1-(r / R))^{1-n}}
$$

Then, according to Eq. (36), we obtain for Eq. (35):

$$
\left(\frac{d u}{d r}\right)_{r=0}=0
$$

Respectively, according to Eq. (37), we obtain for Eq. (31):

$$
\left(\frac{d u}{d r}\right)_{r=0}=-\frac{f(0) \cdot n}{R}
$$

(2) Equation (31), in contrast to Eq. (29), unites all motion regimes, including the laminar motion.

Proceeding from Eq. (24), we can find that the radius forming the separation coefficient is given by:

$$
\frac{r_{0}}{R}=\left[1-\frac{n}{n+2} \cdot \sqrt{\frac{4 B}{3 \lambda}}\right]^{1 / n}
$$


Hence, we obtain a relationship for the distribution coefficient:

$$
K=\left[1-\frac{n}{n+2} \sqrt{\frac{4 B}{3 \lambda}}\right]^{2 / n}
$$

\section{LAMINAR OVERFLOW REGIME}

In this case, the medium motion regime in the channel is based on the dependence of the flow structure expressed by Eq. (35). Thus, in the single-phase (pure) flows at $n=2$, a parabolic velocity profile of a laminar flow takes place, turbulent motion at $n>8$, and a transitory mode at $2<n<8$.

Then according to Eq. (24), we obtain:

$$
\frac{n+2}{n}\left[1-\left(\frac{r_{0}}{R}\right)^{n}\right]=\sqrt{\frac{4 B}{3 \lambda}}
$$

The drag coefficient in the turbulent region is constant and its value is $\lambda=0,5$. In the laminar region, the drag coefficient is inversely proportional to the number $\operatorname{Re}_{\rho}$. A known interpolation formula for the entire overflow velocity region has the form:

$$
\lambda=\frac{24}{\operatorname{Re}_{\rho}}+0,5
$$

At low $\operatorname{Re}_{\rho}$ values, the first term in Eq. (43) is dominating, while at high values, the second term is important. The laminar region is limited by $\operatorname{Re}_{\rho} \leq 1$, and turbulent the region by $\operatorname{Re}_{\rho} \geq 500$. This dependence, as is well-known, also describes well the changes in the drag coefficient in the transitory region of the overflow of particles at $1 \leq \operatorname{Re}_{\rho} \leq 500$.

We can obtain, from Eq. (10):

$$
A r=\frac{3 \lambda}{4} \mathrm{Re}_{\rho}^{2}
$$

By substituting the general value of $\lambda$ into this expression:

$$
A r=\frac{3}{4}\left(\frac{24}{\operatorname{Re}_{\rho}}+0,5\right) \operatorname{Re}_{\rho}^{2}=18 \operatorname{Re}_{\rho}+\frac{3}{8} \operatorname{Re}_{\rho}^{2}
$$

we obtain a quadratic equation with one root already known. For the turbulent mode at $\lambda=0,5$ :

$$
A r=\frac{3}{8} \mathrm{Re}_{\rho}^{2}
$$


Hence:

$$
\operatorname{Re}_{\rho}=\sqrt{\frac{8}{3} A r}
$$

We can linearize the quadratic equation, and we find its second root:

$$
A r=18 \operatorname{Re}_{\rho}+\frac{3}{8} \operatorname{Re}_{\rho} \cdot \sqrt{\frac{8}{3} A r}
$$

Hence, for all overflow regimes:

$$
\operatorname{Re}_{\rho}=\frac{A r}{18+0,61 \sqrt{A r}}
$$

and we can obtain:

$$
\lambda=\frac{4}{3} \frac{(18+0,61 \sqrt{A r})^{2}}{A r}
$$

It then follows from Eq. (42) that:

$$
\frac{n+2}{n}\left[1-\left(\frac{r_{0}}{R}\right)^{n}\right]=\frac{\sqrt{A r \cdot B}}{18+0,61 \sqrt{A r}}
$$

Since the $A r$ value is not high in the laminar mode, Eq. (51) can be simplified and reduced, with high precision, to:

$$
\frac{n+2}{n}\left[1-\left(\frac{r_{0}}{R}\right)^{n}\right]=\frac{\sqrt{A r \cdot B}}{18}
$$

Taking into account the fact that $\left(r_{0} / R\right)=K$ for a parabolic profile $(n=2)$, we finally obtain:

$$
K=1-\frac{\sqrt{A r \cdot B}}{36}
$$

In a general case, this parameter is determined by the expression:

$$
K=\left[1-\frac{n}{n+2} \frac{\sqrt{A r \cdot B}}{(18+0,61 \sqrt{A r})}\right]^{2 / n}
$$

As a result of numerous investigations [1,2], it has been established that in a two-phase flow in the transitory and the turbulent modes, the flow profile is somewhat stretched 
in its central area and approaches a parabolic profile. Therefore, under certain assumptions, $n=2$ can be accepted for all regimes.

Furthermore, for an arbitrary mode of particle overflow, the following relationship can be written:

$$
K=\left(1-\frac{\sqrt{A r \cdot B}}{36+1,22 \sqrt{A r}}\right)
$$

In the turbulent regimes, $A r$ acquires high values; therefore the first term in the denominator can be neglected, and for these regimes, we thus have:

$$
K=(1-\sqrt{0,4 B})
$$

An interesting conclusion can be inferred from Eq. (56). Its generalizing parameter is unambiguously connected with the separation coefficient $K$ determined by the flow structure. Proceeding from this expression or, analogously, from Eqs. (53) and (55), a novel criteria for gravitational classification can be formulated:

for turbulent overflow regimes:

$$
H_{1}=1-K=\sqrt{0,4 B}
$$

for laminar overflow:

$$
H_{2}=\frac{\sqrt{A r \cdot B}}{36},
$$

for arbitrary regimes of particles overflow:

$$
H_{3}=\frac{\sqrt{A r \cdot B}}{36+1,22 \sqrt{A r}}
$$

\section{THE GENERALIZING CRITERIA}

The criteria obtained here can be used for the processing of the experimental data presented in the first part of the paper. The experimental data on separation of aluminum powder, shown in Fig. 3 and evaluated using Eq. (58), result in the dependence presented in Fig. 6, and those evaluated using Eq. (57) in the dependence depicted in Fig. 7. The separation of chromium oxide is shown in Fig. 2 and evaluated using Eq. (58) is shown in Fig. 8. It follows from these examples that all these criteria provide a generalizing effect.

Now we can return to the dependence in Eq. (58). It is clear that the value 36 in the denominator represents a scaling factor, whereas the physical meaning of the parameter is in the numerator. Let us analyse it:

$$
\sqrt{A r \cdot B}=\frac{q d^{2}\left(\rho-\rho_{0}\right)}{\mu \cdot w}
$$




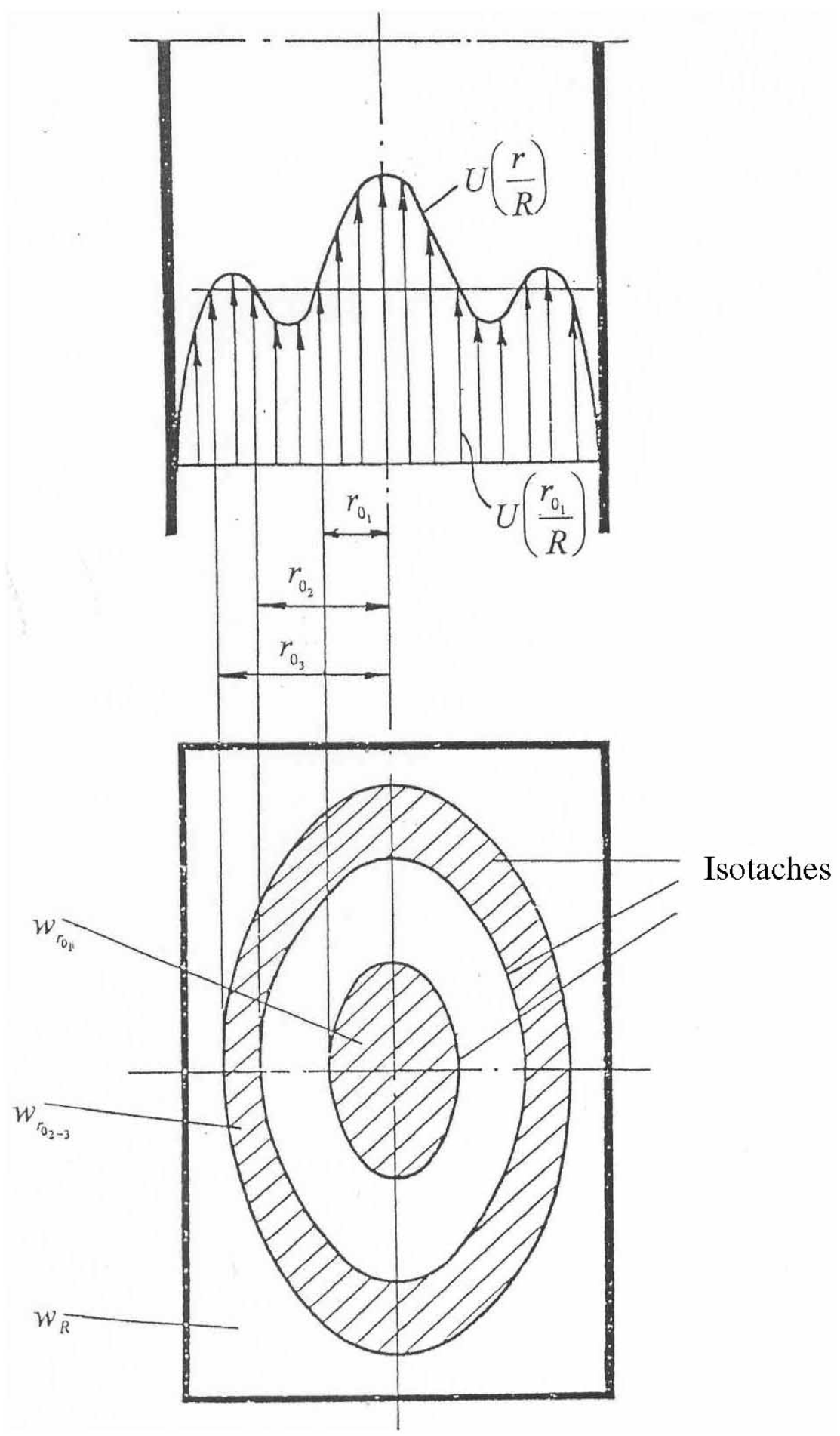

FIGURE 5 Formation of the distribution coefficient in case of a complicated profile of a continuous medium. 


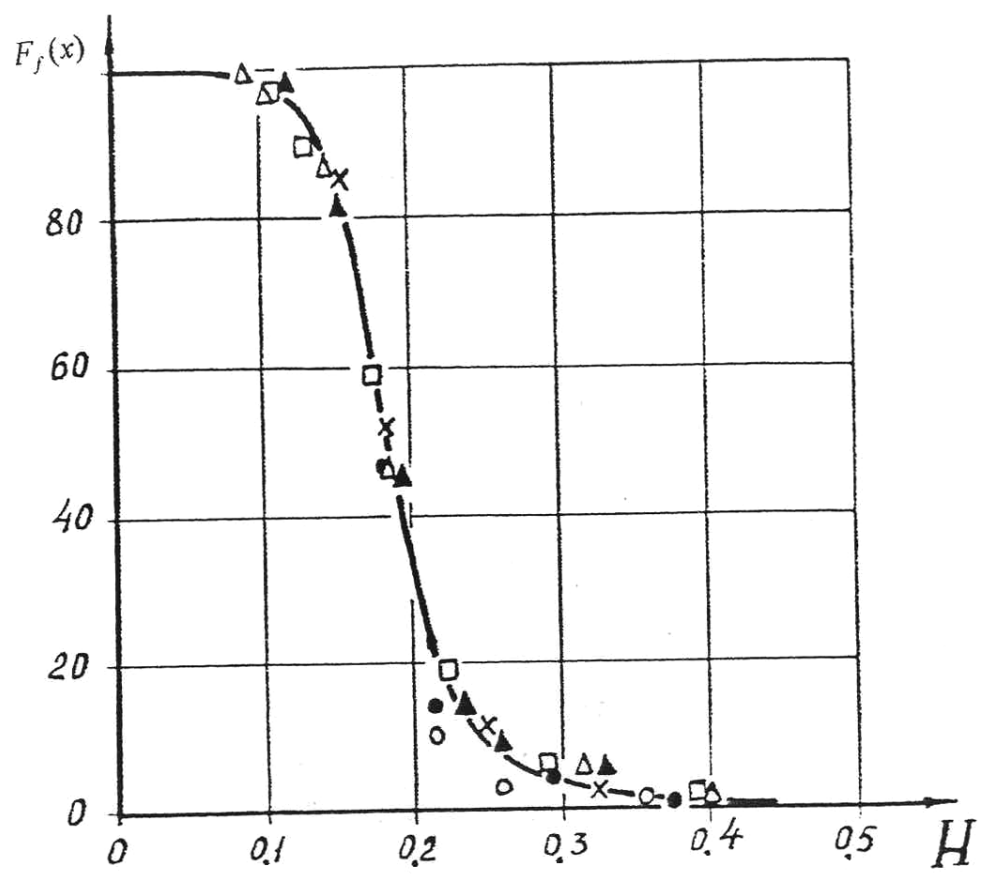

FIGURE 6 Dependence of $F_{f}(x)=f(H)$ type for aluminum powder. Notation of the velocities: $0-0.27 \mathrm{~m} / \mathrm{s}$; - $-0.36 \mathrm{~m} / \mathrm{s} ; \times-0.51 \mathrm{~m} / \mathrm{s} ; \square-0.9 \mathrm{~m} / \mathrm{s} ; \Delta-1.32 \mathrm{~m} / \mathrm{s} ; \boldsymbol{\Delta}-1.7 \mathrm{~m} / \mathrm{s}$.

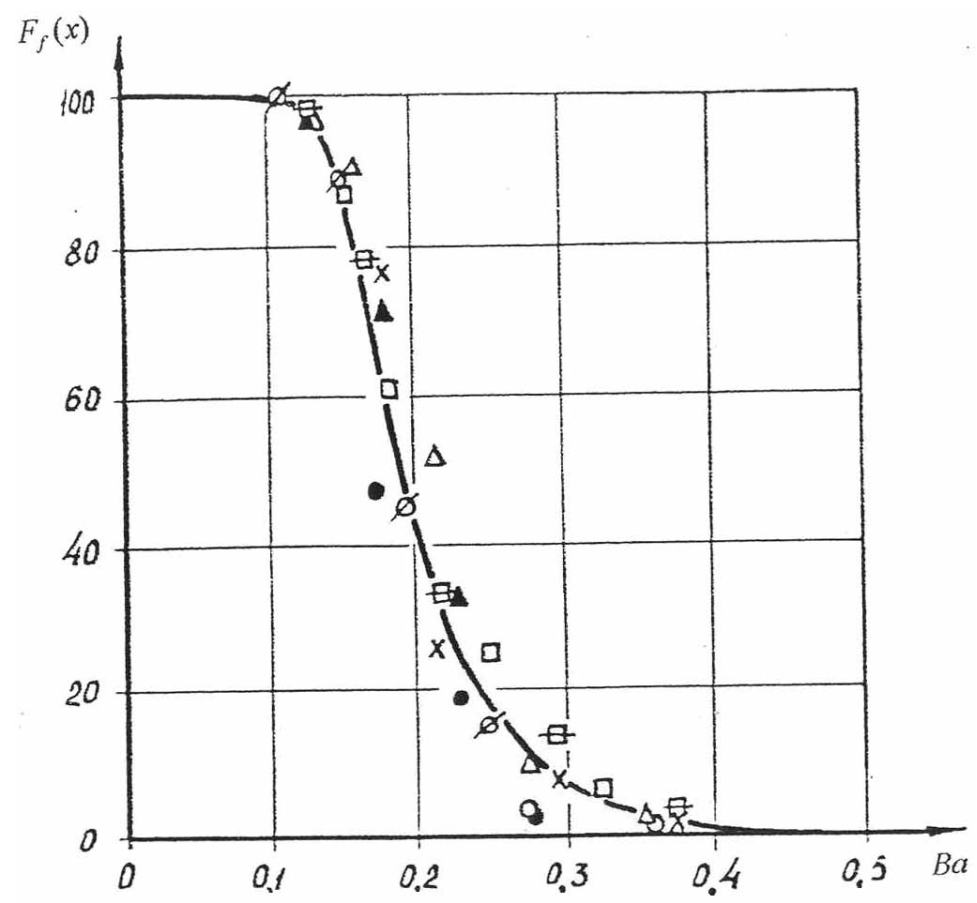

FIGURE 7 Dependence of $F_{f}(x)=f(B a)$ type for aluminum powder. For notation, see Fig. 6. 


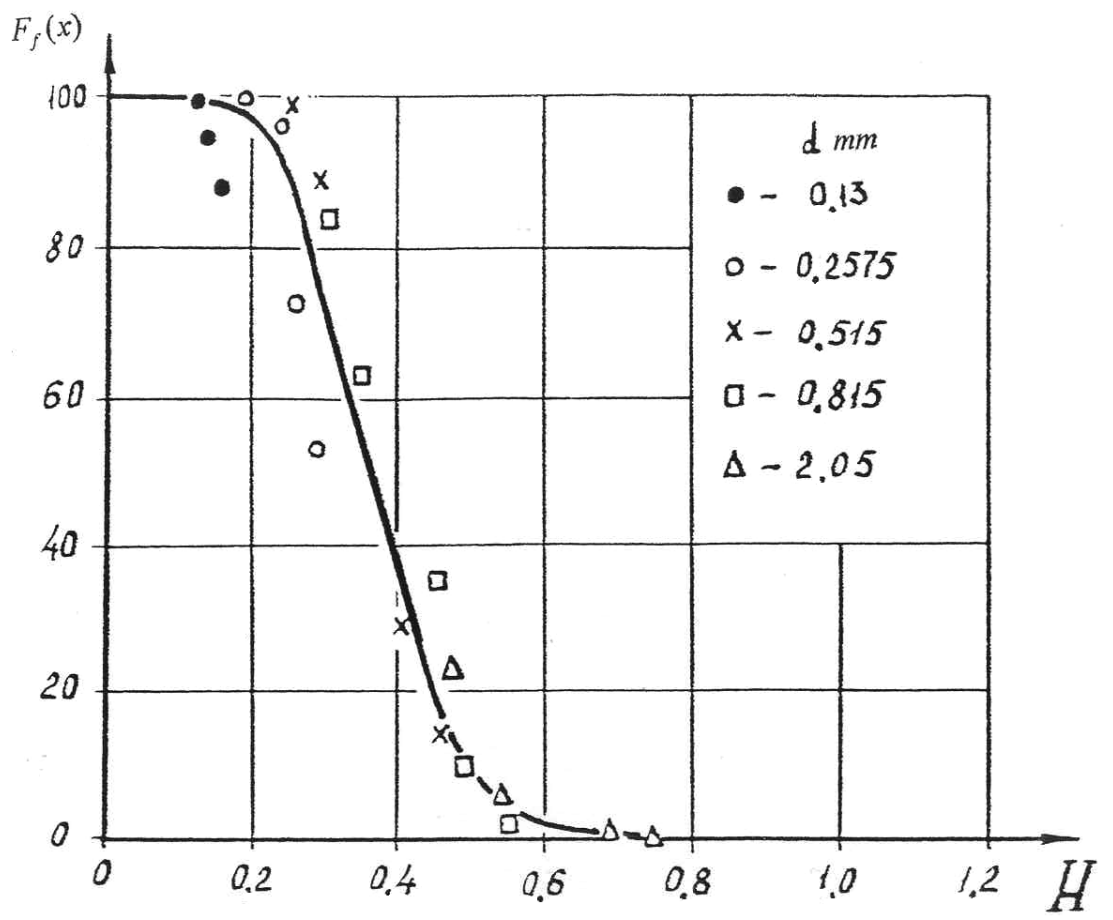

FIGURE 8 Dependence of $F_{f}(x)=f(H)$ type for chromium oxide. Notation of the mean particle size: $0.13 \mathrm{~mm}$; O $-0.258 \mathrm{~mm} ; \times-0.515 \mathrm{~mm} ; \square-0.815 \mathrm{~mm} ; \Delta-2.05 \mathrm{~mm}$.

A new dimensionless criterion valid for laminar overflow regimes can thus be obtained. These regimes cover most wet separation processes and also dry separation of very fine particles.

Thus, we have formulated generalizing parameters that make it possible to obtain universal separation curves:

- at a turbulent overflow of particles:

$$
B=\frac{q d}{w^{2}} \frac{\left(\rho-\rho_{0}\right)}{\rho_{0}},
$$

- at a laminar overflow of particles:

$$
B_{a}=\frac{q d^{2}\left(\rho-\rho_{0}\right)}{\mu w}
$$

- at any overflow regimes:

$$
H=\frac{\sqrt{A r \cdot B}}{36+1,22 \sqrt{A r}}
$$


Let us analyze the relationships between $B$ and $B_{a}$ criteria. For this purpose, their ratio can be written as:

$$
\frac{B_{a}}{B}=\frac{q d^{2}\left(\rho-\rho_{0}\right) w^{2} \rho_{0}}{\mu \cdot w \cdot q d\left(\rho-\rho_{0}\right)}=\frac{d w \rho_{0}}{\mu}=\mathrm{Re}
$$

This ratio gives the value of the Reynolds number determined for a particle. Thus, we can write:

$$
B_{a}=B \cdot \operatorname{Re}
$$

This relationship has a fundamental physical meaning. In the modes of turbulent overflow of particles, the Reynolds number degenerates, and then the criterion $B$ is sufficient. In the laminar region, where the drag on the particles is proportional to $\mathrm{Re}$, the product $B \cdot \mathrm{Re}$ is required for the generalization of the separation curves.

\section{References}

[1] E. Barsky and M. Barsky, Master curve of separation processes. Phys. Sep. Sci. Engin., 13 (2004), 1-13.

[2] M.D. Barsky, Powder Fractionation, Moscow, Nedra, 1980 (in Russian).

[3] E. Barsky and M. Buikis, Mathematical model for gravitational cascade separation of pourable materials at identical stages of the classifier. Progress in Industrial Mathematics, Springer, 2004, pp. 229-233.

[4] V.G. Ainshtein, General Course of Chemical Technology Processes and Apparatuse, Moscow, Logos, Higher School, 2002 (in Russian). 